\title{
Assessing Psychological Response to the COVID-19: The Fear of COVID-19 Scale and the COVID Stress Scales
}

\author{
Amir H. Pakpour ${ }^{1}$ - Mark D. Griffiths ${ }^{2} \cdot$ Chung-Ying Lin $^{3}$ (D \\ Published online: 29 May 2020 \\ (C) Springer Science+Business Media, LLC, part of Springer Nature 2020
}

The threat of novel coronavirus disease 2019 (COVID-19) has been well acknowledged and its impacts on individuals' psychological responses have been empirically reported (e.g., Bhuiyan et al. 2020; Qiu et al. 2020; Wang et al. 2020). Such psychological responses, especially anxiety and fear, are important factors for an individual's behaviors. Taylor (2019) indicated that low levels of anxiety may lead to reluctant prevention behaviors (e.g., handwashing) and high level of anxiety may lead to socially disruptive behaviors (e.g., panic buying) (Taylor et al. 2020; Lin 2020). Therefore, there is a need for healthcare providers to have a valid instrument to assess an individual's psychological responses specifically toward COVID-19 during the pandemic (Taylor et al. 2020).

In this regard, Taylor et al. (2020) and Ahorsu et al. (2020) developed different instruments to understand such psychological responses. More specifically, Taylor et al. (2020) developed the COVID Stress Scales (CSS) and we developed the Fear of COVID-19 Scale (FCV-19S; Ahorsu et al. 2020). The CSS was developed using representative samples from Canada $(n=$ $3479)$ and the USA ( $n=3375$; Taylor et al. 2020), whereas the FCV-19S was developed using a sample from Iran ( $n=717$; Ahorsu et al. 2020). The FCV-19S has since been translated and validated in different languages including Turkish ( $n=1304$; Satici et al. 2020), Arabic $(n=$ 639; Alyami et al. 2020), Italian ( $n=250$; Soraci et al. 2020), Russian $(n=800$; Reznik et al. 2020), Hebrew ( $n=639$; Bitan et al. 2020), and Bangla ( $n=8550$; Sakib et al. 2020).

In Taylor et al.'s (2020) study, the CSS was found to be valid and reliable. The CSS was firstly tested on the Canadian sample after its items had been generated using a standard method to develop a new instrument. The CSS was then replicated utilizing an American sample. Parallel analysis and exploratory factor analysis were firstly performed using the Canadian sample for the CSS and a five-factor structure (COVID danger and contamination

Amir H. Pakpour and Mark D. Griffiths contributed equally to this work.

Chung-Ying Lin

cylin36933@gmail.com; cy.lin@polyu.edu.hk

1 Department of Nursing, School of Health and Welfare, Jönköping University, Jönköping, Sweden

2 International Gaming Research Unit, Psychology Department, Nottingham Trent University, Nottingham, UK

3 Department of Rehabilitation Sciences, Faculty of Health and Social Sciences, The Hong Kong Polytechnic University, 11 Yuk Choi Rd, Hung Hom, Hong Kong 
fears; COVID fears on economic consequences; COVID xenophobia; COVID compulsive checking; and COVID traumatic stress symptoms) was identified. The five-factor structure of the CSS was later confirmed in the American sample using confirmatory factor analysis. Apart from the structure, internal consistency, convergent validity, and discriminant validity of the CSS were also supported in the Taylor et al.'s (2020) study.

Although we appreciate that Taylor et al. (2020) developed a new instrument assessing stress- and anxiety-related symptoms toward COVID-19, we would like to specifically respond to their comment concerning the FCV-19S (p. 5): “... while several measures of COVID-19-related fears have recently emerged (e.g., Ahorsu et al. 2020), they tend to be unidimensional (i.e., focused on general fear aspects of COVID-19) and are based on limited psychometric evaluation." More specifically, there are two points we wish to take issue with: (i) the implied criticism that unidimensional nature of the FCV-19S is a weakness, and (ii) the claim that our scale underwent only limited psychometric evaluation.

Regarding the unidimensional nature of the FCV-19S, there is no consensus in the psychometric literature as to whether instruments that have unidimensional structure or a multidimensional structure outperform one another. Psychometric instruments having unidimensional and multidimensional structures have been concluded as having different features and benefits. For example, the US Food and Drug Administration (FDA) released a report to guide the use of patient-reported outcome (PRO) measures, which are usually psychometric instruments (e.g., depression inventories, quality of life scales), in relation to drug effects, and the report only supported the use of unidimensional PRO measures (FDA 2009). One strength of using unidimensional instruments is their simplicity (i.e., the underlying construct of the specific instrument is straightforward). However, we acknowledge that multidimensional instruments sometimes have the benefits of providing more holistic results than unidimensional instruments (Lin et al. 2017). In short, there are benefits of both unidimensional and multidimensional instruments for COVID-19 (e.g., the FCV-19S [Ahorsu et al. 2020] and the CSS [Taylor et al. 2020]).

Regarding the psychometric evaluation of the FCV-19S, we acknowledge that the first study examining the properties the FCV-19S (Ahorsu et al. 2020) did not carry out confirmatory factor analysis. However, our aim was to quickly develop a psychometrically validated instrument for healthcare providers to use during a time of urgent need. Furthermore, although advanced psychometric testing of confirmatory factor analysis was not performed in our first FCV-19S study, another advanced psychometric model (i.e., Rasch analysis) was carried out. Rasch analysis includes the following features that supplement to the findings from a classical test theory model (e.g., confirmatory factor analysis). First, the factor structure of an instrument is intertwined with the difficulty of the questions. However, the item difficulties may perform differently among different populations. For example, mental health professionals as compared with people with depression may tend to score higher in mental health questionnaires because mental health professionals have more skills to cope with distress. Therefore, item difficulty and individual ability should be separately estimated. Therefore, Rasch analysis is an appropriate testing method to separately estimate item difficulty and individual ability. Second, because most instruments are assessed using a Likert scale (i.e., an ordinal scale), the relationship between instruments' item scores and underlying factor scores may be nonlinear. Given that the Rasch model converts the ordinal scales into probability (i.e., a ratio scale), the nonlinear relationship between item scores and factor scores can be resolved (Chang et al. 2015). 
Moreover, we asserted in our original validation study that future studies should further examine the psychometric properties of the FCV-19S to provide additional scientific rigor and cumulative evidence is needed for every newly developed psychometric instrument (Lin et al. 2019). Since the initial publication of the FCV-19S, the instrument has been validated in different language versions using various types of psychometric testing, including confirmatory factor analysis, Rasch analysis, concurrent validity testing, internal consistency, and test-retest reliability (Ahorsu et al. 2020; Sakib et al. 2020; Satici et al. 2020; Soraci et al. 2020). We have been approached by research teams in over 20 different countries to validate the FCV-19S, and we are aware of a number of studies currently under review that have also found the FCV-19S to have excellent psychometric properties. Furthermore, the efficacy of the FCV-19S has been demonstrated by its strong association with relevant human behaviors, such as preventive behaviors of spatial distancing and hand hygiene behaviors (Harper et al. 2020; Pakpour and Griffiths 2020). Therefore, we are confident that the FCV-19S is a robust instrument to assess fear of COVID-19 and that its psychometric properties are not limited as claimed by Taylor et al. (2020).

To conclude, we believe that both the CSS and FCV-19S are potential useful instruments that can be used by healthcare providers to help understand the psychological responses such as fear and anxiety of individuals during the COVID-19 pandemic. However, healthcare providers may consider different purposes to select a suitable instrument (either the CSS or the FCV-19S). For example, the CSS may provide comprehensive information regarding stress and anxiety of an individual because of its multidimensional structure. However, the CSS takes a much longer time to complete than the FCV-19S because the FCV-19S only contains seven items. Consequently, the FCV-19S may be especially useful in a busy clinical setting. Moreover, the FCV-19S has the advantage of different language versions, including English (Harper et al. 2020), Persian (Ahorsu et al. 2020), Bangla (Sakib et al. 2020), Italian (Soraci et al. 2020), Hebrew (Bitan et al. 2020), Arabic (Alyami et al. 2020), Russian (Reznik et al. 2020), and Turkish (Satici et al. 2020), and already has the potential to be used for country comparisons.

\section{Compliance with Ethical Standards}

Conflict of Interest The authors declare that they have no conflict of interest.

\section{References}

Ahorsu, D. K., Lin, C. Y., Imani, V., Saffari, M., Griffiths, M. D., \& Pakpour, A. H. (2020). The fear of COVID19 scale: Development and initial validation. International Journal of Mental Health and Addiction. Advance online publication. https://doi.org/10.1007/s11469-020-00270-8.

Alyami, M., Henning, M., Krägeloh, C. U., \& Alyami, H. (2020). Psychometric evaluation of the Arabic version of the Fear of COVID-19 Scale. International Journal of Mental Health and Addiction. Advance online publication. https://doi.org/10.1007/s11469-020-00316-x.

Bitan, D. T., Grossman-Giron, A., Bloch, Y., Mayer, Y., Shiffman, N., \& Mendlovic, S. (2020). Fear of COVID19 scale: Psychometric characteristics, reliability and validity in the Israeli population. Psychiatry Research. Advance online publication, 289, 113100. https://doi.org/10.1016/j.psychres.2020.113100.

Bhuiyan, A. I., Sakib, N., Pakpour, A. H., Griffiths, M. D., \& Mamun, M. A. (2020). COVID-19-related suicides in Bangladesh due to lockdown and economic factors: Case study evidence. International Journal of Mental Health and Addiction. Advance online publication. https://doi.org/10.1007/s11469-020-00307-y.

Chang, C.-C., Su, J.-A., Tsai, C.-S., Yen, C.-F., Liu, J.-H., \& Lin, C.-Y. (2015). Rasch analysis suggested three unidimensional domains for Affiliate Stigma Scale: Additional psychometric evaluation. Journal of Clinical Epidemiology, 68(6), 674-683. https://doi.org/10.1016/j.jclinepi.2015.01.018. 
Harper, C. A., Satchell, L. P., Fido, D., \& Latzman, R. D. (2020). Functional fear predicts public health compliance in the COVID-19 pandemic. International Journal of Mental Health and Addiction. Advance online publication. https://doi.org/10.1007/s11469-020-00281-5.

Lin, C.-Y. (2020). Social reaction toward the 2019 novel coronavirus (COVID-19). Social Health and Behavior, 3, 1-2. https://doi.org/10.4103/SHB.SHB_11_20.

Lin, C.-Y., Hwang, J.-S., Wang, W.-C., Lai, W.-W., Su, W.-C., Wu, T.-Y., Yao, G., \& Wang, J.-D. (2019). Psychometric evaluation of the WHOQOL-BREF, Taiwan version, across five kinds of Taiwanese cancer survivors: Rasch analysis and confirmatory factor analysis. Journal of the Formosan Medical Association, 118, 215-222. https://doi.org/10.1016/j.jfma.2018.03.018.

Lin, C.-Y., Wang, J.-D., Pai, M.-C., \& Ku, L.-J. E. (2017). Measuring burden in dementia caregivers: Confirmatory factor analysis for short forms of the Zarit burden interview. Archives of Gerontology and Geriatrics, 68, 8-13. https://doi.org/10.1016/j.archger.2016.08.005.

Pakpour, A. H., \& Griffiths, M. D. (2020). The fear of COVID-19 and its role in preventive behaviors. Journal of Concurrent Disorders, 2(1), 58-63.

Reznik, A., Gritsenko, V., Konstantinov, V., Khamenka, N., \& Isralowitz, R. (2020). COVID-19 fear in Eastern Europe: Validation of the Fear of COVID-19 Scale. International Journal of Mental Health and Addiction. Advance online publication. https://doi.org/10.1007/s11469-020-00283-3.

Qiu, J., Shen, B., Zhao, M., Wang, Z., Xie, B., \& Xu, Y. (2020). A nationwide survey of psychological distress among Chinese people in the COVID-19 epidemic: Implications and policy recommendations. General Psychiatry, 33, e100213. https://doi.org/10.1136/gpsych-2020-100213.

Sakib, N., Mamun, M. A., Bhuiyan, A. K. M. I., Hossain, S., Mamun, F. A., Hosen, I., et al. (2020). Psychometric validation of the Bangla Fear of COVID-19 Scale: Confirmatory factor analysis and Rasch analysis. International Journal of Mental Health and Addiction. Advance online publication. https://oi. org/10.1007/s11469-020-00289-x.

Satici, B., Gocet-Tekin, E., Deniz, M. E., \& Satici, S. A. (2020). Adaptation of the Fear of COVID-19 Scale: Its association with psychological distress and life satisfaction in Turkey. International Journal of Mental Health Addiction. Advance online publication. https://doi.org/10.1007/s11469-020-00294-0.

Soraci, P., Ferrari, A., Abbiati, F. A., Del Fante, E., De Pace, R., Griffiths, U. A., \& M.D. (2020). Validation and psychometric evaluation of the Italian version of the Fear of COVID-19 Scale. International Journal of Mental Health and Addiction. Advance online publication. https://doi.org/10.1007/s11469-020-00277-1.

Taylor, S. (2019). The psychology of pandemics: Preparing for the next global outbreak of infectious disease. Newcastle upon Tyne, UK: Cambridge Scholars Publishing.

Taylor, S., Landry, C., Paluszek, M., Fergus, T. A., Mckay, D., \& Asmundson, G. J. G. (2020). Development and initial validation of the COVID Stress Scales. Journal of Anxiety Disorders, 72, 102232. https://doi. org/10.1016/j.janxdis.2020.102232.

The US Food and Drug Administration. (2009). Patient-reported outcome measures: Use in medical product development to support labeling claims. Retrieved from https:/www.fda.gov/regulatory-information/searchfda-guidance-documents/patient-reported-outcome-measures-use-medical-product-development-supportlabeling-claims. Accessed May 10, 2020.

Wang, C., Pan, R., Wan, X., Tan, Y., Xu, L., Ho, C. S., \& Ho, R. C. (2020). Immediate psychological responses and associated factors during the initial stage of the 2019 coronavirus disease (COVID-19) epidemic among the general population in China. International Journal of Environmental Research and Public Health, 17, 1729. https://doi.org/10.3390/ijerph17051729.

Publisher's Note Springer Nature remains neutral with regard to jurisdictional claims in published maps and institutional affiliations. 